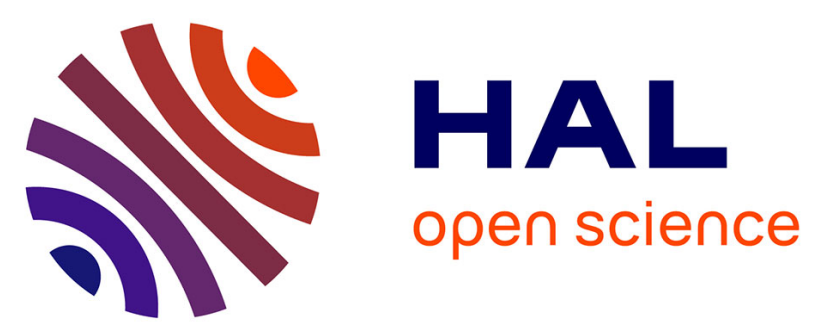

\title{
Contrast-to-Tissue Ratio Improvement by Transmitted Optimized Binary Signal in Ultrasound Pulse Inversion Imaging
}

Sébastien Ménigot, Jean-Marc Girault

\section{- To cite this version:}

Sébastien Ménigot, Jean-Marc Girault. Contrast-to-Tissue Ratio Improvement by Transmitted Optimized Binary Signal in Ultrasound Pulse Inversion Imaging. 2013 IEEE 10th International Symposium on Biomedical Imaging (ISBI), Apr 2013, San Francisco, United States. pp.776-779, 10.1109/ISBI.2013.6556590 . hal-00881440

\section{HAL Id: hal-00881440 \\ https://hal.science/hal-00881440}

Submitted on 8 Nov 2013

HAL is a multi-disciplinary open access archive for the deposit and dissemination of scientific research documents, whether they are published or not. The documents may come from teaching and research institutions in France or abroad, or from public or private research centers.
L'archive ouverte pluridisciplinaire $\mathbf{H A L}$, est destinée au dépôt et à la diffusion de documents scientifiques de niveau recherche, publiés ou non, émanant des établissements d'enseignement et de recherche français ou étrangers, des laboratoires publics ou privés. 


\title{
CONTRAST-TO-TISSUE RATIO IMPROVEMENT BY TRANSMITTED OPTIMIZED BINARY SIGNAL IN ULTRASOUND PULSE INVERSION IMAGING
}

\author{
Sébastien Ménigot ${ }^{\star \dagger}$ and Jean-Marc Girault ${ }^{\dagger \ddagger}$ \\ * IUT Ville d'Avray, université Paris Ouest Nanterre La Défense, Ville d'Avray, France \\ $\dagger$ UMR-S930, université François-Rabelais de Tours, Tours, France \\ $\ddagger$ U 930, Inserm, Tours, France
}

C2013 IEEE. Reprinted, with permission, from Sébastien Ménigot and Jean-Marc Girault, Contrast-to-Tissue Ratio Improvement by Transmitted Optimized Binary Signal in Ultrasound Pulse Inversion 2013. This material is posted here with permission of the IEEE. Such permission of the IEEE does not in any way imply IEEE endorsement of any of the Université François Rabelais de Tours' products or services. Internal or personal use of this material is permitted. However, permission to reprint/republish this material for advertising or promotional purposes or for creating new collective works for resale or redistribution must be obtained from the IEEE by writing to pubs-permissions@ieee.org.

DOI : 10.1109/ISBI.2013.6556590

\begin{abstract}
Ultrasound contrast imaging has provided more accurate medical diagnoses. One of the most used techniques is the pulse inversion imaging improving the contrast-to-tissue ratio $(C T R)$ by extracting nonlinearities of contrast agents. The usual transmitted signal is at a fixed frequency. However, an optimal choice requires information about the transducer and the medium. This information is experimentally inaccessible. Moreover, the digital electronic setup can limit the solution. Our goal was to seek the binary command which maximized the CTR. A genetic algorithm sought the vector of input binary samples. By adding a closed loop, the system automatically proposed the optimal binary command without any a priori information about the system or the medium explored and without hypothesis on binary samples. In simulation, the gain compared with a transmitted signal at the optimal frequency can reach about $3 \mathrm{~dB}$ and $0.5 \mathrm{~dB}$ in comparison with a transmitted signal at the two-thirds of the central frequency of the transducer.
\end{abstract}

Index Terms - Binary Signal, Genetic Algorithm, Optimal Command, Pulse Inversion, Ultrasound Imaging

\section{INTRODUCTION}

Since the 1960s, medical ultrasound imaging has become an essential tool for clinical diagnosis. Particularly, intravenous injection of ultrasound contrast agents containing microbubbles have enabled to extract physiological and pathological information [1]. In this context of ultrasound contrast imaging, improvements have been led thanks to the nonlinear behavior of ultrasound contrast agents. However, these improvements have been limited because of the effects of the ultrasound wave propagation. The nonlinear components generated in tissue reduce the contrast-to-tissue ratio $(C T R)$. Moreover, the axial resolution has been limited, because good separation of the harmonic components requires a limited pulse bandwidth, as in second harmonic imaging [1].

Several imaging methods have been proposed to improve contrast by using the nonlinearities, such as second harmonic imaging [1], subharmonic imaging [2], super harmonic imaging [3]. Hovewer, to ensure a good axial resolution while increasing the contrast, some techniques have been based on discrete encoding, such as pulse inversion imaging [4], power modulation [5], contrast pulse sequencing [6] or pulse subtraction [7]. Other imaging methods are based on continous encoding, such as harmonic chirp imaging [8] to solve the trade-off between resolution and penetration. Since the most commonly used is the pulse inversion imaging, we only focused our study with this technique.

To optimally use the pulse inversion imaging, the transmitted pulse must be correctly adjusted. The problem was to find the optimal command $w^{\star}(t)$ of the pulse inversion imaging system which provides the best $C T R$ :

$$
w^{\star}(t)=\arg \max _{w(t)}(C T R(w(t))),
$$

Conventional ultrasound scanners can usually provide up to three transmit frequencies for manual selection to construct a transmitted signal at this fixed frequency. Nowadays, any method can solve satisfactorily and optimally this problem. Time reversal imaging has enable to minimize the tissue backscattering to improve the CTR [9], but without taking into account microbubble backscattering. However, an analytic solution has been proposed [10]. Unfortunately, the solution of the problem requires (i) inaccessible a priori knowledges of the medium and the transducer and (ii) hard solver implementation. The second solution carries on transforming the 
shape optimization in a suboptimal parametric optimization; for example the transmit frequency [11]. Although the optimal waveform found by the first solution is nonlinear and thus a transmit frequency parameter is not enough to describe the waveform, these techniques have shown that it was important to find the optimal command to maximize the CTR.

Unfortunately, commercial ultrasound devices have only unipolar or bipolar ultrasound transmitter, which do not enable a direct application of the previous methods. Several imaging methods improved the signal-to-noise ratio [12] or the microbubble detection [13] by combining a binary waveform and an advanced imaging approach. Nevertheless, no input optimization process was dedicated to find the binary optimal command.

The aim of the study was to find automatically the optimal binary command of the ultrasound pulse inversion imaging system to provide the best CTR. We therefore replaced the current system with a closed loop system whose transmitted pulse was modified by feedback. We propose to solve the shape optimization by using a genetic algorithm and we applied it in simulation. The advantage of the method was the optimization without a priori knowledge in order to find the optimal binary shape.

\section{METHODS}

The principle of pulse inversion imaging including feedback is described in Fig. 1. For an individual solution at the iteration $k$, two binary pulses $x_{k, 1}(n)$ and $x_{k, 2}(n)$ with opposite phase were transmitted. The sum $z_{k}(n)$ of the two respective echoes $y_{k, 1}(n)$ and $y_{k, 2}(n)$ formed a radiofrequency line $l_{k}$. From the $C T R_{k}$ estimated on this radiofrequency line $l_{k}$, a new transmitted binary signal $x_{k+1}(n)$ was computed by the algorithm to optimize the $C T R_{k+1}$.

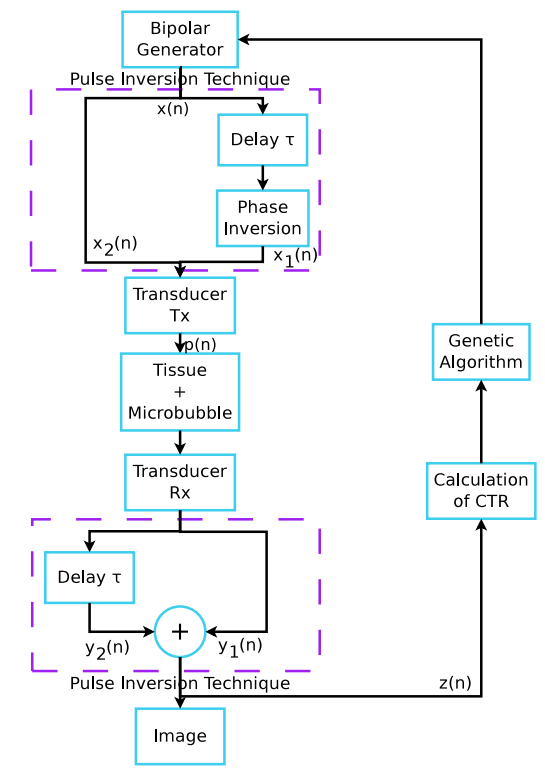

Fig. 1. Block diagram of CTR optimization in pulse inversion imaging.

\subsection{Binary Transmitted Signal}

The pulse signal $x_{k, q}(n)$ was digitally computed with Matlab (Mathworks, Natick, MA, USA):

$$
x_{k, q}(n)=\left\{\begin{array}{lll}
A \cdot w_{k}(n) & \text { if } & q=1 \\
A \cdot\left(1-w_{k}(n)\right) & \text { if } & q=2
\end{array}\right.
$$

where $n$ is the discrete time. The binary signal $w_{k}(n)$ was defined on a duration $T$, which corresponded to $100 \%$ of the fractional bandwidth of the transducer. The binary signal $w_{k}(n)$ was thus built by $N_{s}$ samples. Their value should be selected for optimization. Note that this number must be sufficient because of the transducer bandwidth, and it must not be too high for the algorithm.

The amplitude of the driving pressure $A$ was then adjusted so that the power of the binary pulse $x_{k, q}(n)$ was constant to $P_{x_{r e f}}$. This power $P_{x_{r e f}}$ was calculated for a signal $x_{r e f}$ at the central frequency $f_{c}$ of the transducer and for the driving pressure $A_{0}$.

\subsection{Cost Function}

Since our aim was to maximize the contrast and since the usual contrast estimator in contrast imaging is the $C T R_{k}$, the cost function was $C T R_{k}$. It is defined as the ratio of the power $P_{b, k}$ backscattered by the area of the perfused medium to the power $P_{t, k}$ backscattered by the area of the non-perfused medium [11]. Note that these powers were computed from the lines $z_{k}(n)$ of the pulse inversion image.

\subsection{Genetic Algorithm}

The metaheuristic can solve hard optimization problem. Among the meta heuristic, genetic algorithm finds the optimum by setting a vector containing parameters. This property led us to use a binary genetic algorithm [14], where the vector was the $N_{s}$ samples of the binary signal $w_{k}(n)$.

The principle is the genetic reproduction in biology, except that each gene can only be 0 or 1 . In our case, at the iteration $k$, a generation $k$ with 12 binary individual solutions (sample vectors) was tested. For the next generation $k+1$, only 6 individual solutions were conserved to become pairs and mates. From these pairs and mates, 6 new individual solutions were made by random cross-over technique. Then $40 \%$ samples were mutated so that the optimization was robust. The best individual solution was the optimal binary command for the generation $k$. Note a small population and a high mutation rate were chosen to solve the trade-off between robustness and the computation time due to sorting of each individual solution [14].

\section{SIMULATION MODEL}

The simulation model was built on the pulse inversion imaging system (Fig. 1). It was composed of different phases: 
transmission, 2D nonlinear propagation, nonlinear oscillations of microbubbles and reception. A pulse wave was propagated nonlinearly into an attenuating medium without microbubbles. This wave, composed of harmonic components, excited a microbubble in the vascular system. The nonlinear oscillations of this microbubble were backscattered and measured by the receiver.

\subsection{Nonlinear Propagation in Tissue}

A binary signal $x_{k, q}(n)$ was generated digitally and filtered by the transfer function of a realistic transducer, centred at $f_{c}=$ $4 \mathrm{MHz}$ with a fractional bandwidth of $75 \%$ at $-3 \mathrm{~dB}$. The 2D nonlinear wave propagation into the medium was obtained by solving Anderson's model based on a pseudo-spectral derivative and a time-domain integration algorithm [15]. The physical parameters used were a density of $928 \mathrm{~kg} \cdot \mathrm{m}^{-3}$, a speed of sound of $1578 \mathrm{~m} \cdot \mathrm{s}^{-1}$, a $B / A$ nonlinearity parameter of 6.7 and an attenuation of $0.45 \mathrm{~dB} \cdot \mathrm{MHz}^{-1.05} \cdot \mathrm{cm}^{-1}$ [16]. Finally, the signal backscaterred by tissue was recorded, whereas the driving pressure at $15 \mathrm{~mm}$ was included into the microbubble model described below.

\subsection{Microbubble}

The simulated ultrasound contrast agent had the properties of encapsulated microbubbles used in clinical practise where the mean diameter was $2.5 \mu \mathrm{m}$ [17] and the resonance frequency was $2.6 \mathrm{MHz}$. The acoustic response was computed for one microbubble from the Marmottant's model [18] based on the Rayleigh-Plesset equation and the polytropic transformation. Finally the echo of the microbubble was deduced from the oscillation. Note that in order to simulate the mean behavior of a microbubble cloud, we hypothesized that the response of a cloud of $N_{b}$ microbubbles was $N_{b}$ times the response of a single microbubble with the mean properties [11]. The microbubble response was thus multiplied in order to simulate a dilution of $1 / 2000$. Moreover, to be more realistic, the attenuation effects due to the high concentration of microbubbles were taken into account [19] for this dilution.

Finally, the echoes from tissue and microbubble were added and filtered by the transfer function of the transducer to build the radiofrequency line $l_{k}$.

\section{RESULTS}

The optimization process was applied in the simulation model to demonstrate the feasibility of our novel method. The driving pressure $A_{0}$ was set to $400 \mathrm{kPa}$. The duration $T$ of the binary signal represented $100 \%$ of the fractional bandwidth of the transducer, i.e. $T=0.3 \mu$ s. The sample number $N_{s}$ was thus 40 according to the sampling rate required to the simulation model. Note that this sampling rate is close to this one in ultrasound imaging.
Fig. 2 shows the best $C T R$ as a function of generation $k$. As an illustration, this result was compared with (i) an usual case where the transmitted signal was at the two-thirds of the central frequency $f_{c}$ of the transducer [20], and (ii) a suboptimal case where the transmitted signal was at the optimal frequency $f_{\text {opt }}[11]$.

After 500 generations, the $C T R$ reached an optimal value superior to the frequency setting cases. The gain reached $3 \mathrm{~dB}$ in comparison with the usual case, and $0.5 \mathrm{~dB}$ in comparison with the case at the optimal frequency $f_{\text {opt }}$.

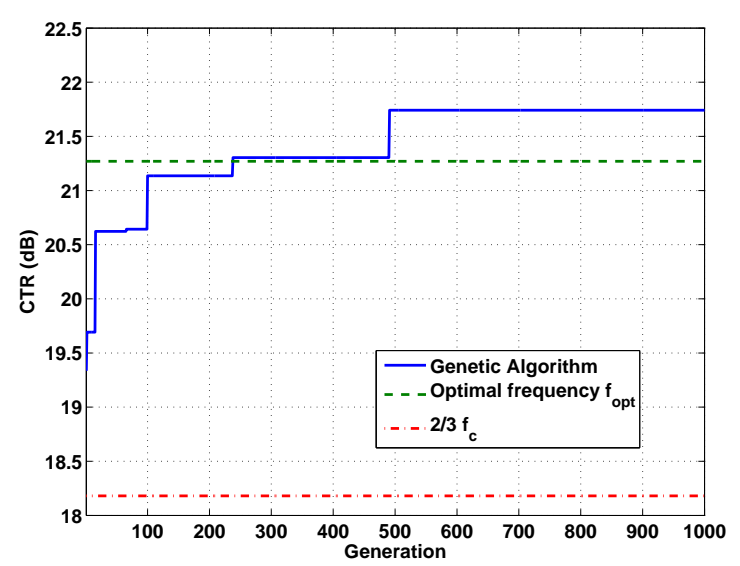

Fig. 2. Simulation of automatic optimization of the contrastto-tissue ratio $(C T R)$ by a binary transmitted signal. The optimization was compared with the cases where the transmitted signal is at the optimal frequency and at the two-thirds of the central frequency of the transducer.

Fig. 3a shows the optimal binary command $w_{\text {opt }}(n)$. The binary signal had not periodicity unlike the usual transmitted signal. As an illustration, Fig. $3 \mathrm{~b}$ shows the signal $p(n)$ at the transucer ouput (Fig. 1) when $w(n)$ was the optimal binary signal (Fig. 3a) and its spectrum in Fig. 3c. This signal was transmitted in tissue. Contrary to the usual transmitted signal, the optimal transmitted signal had nonlinear components.

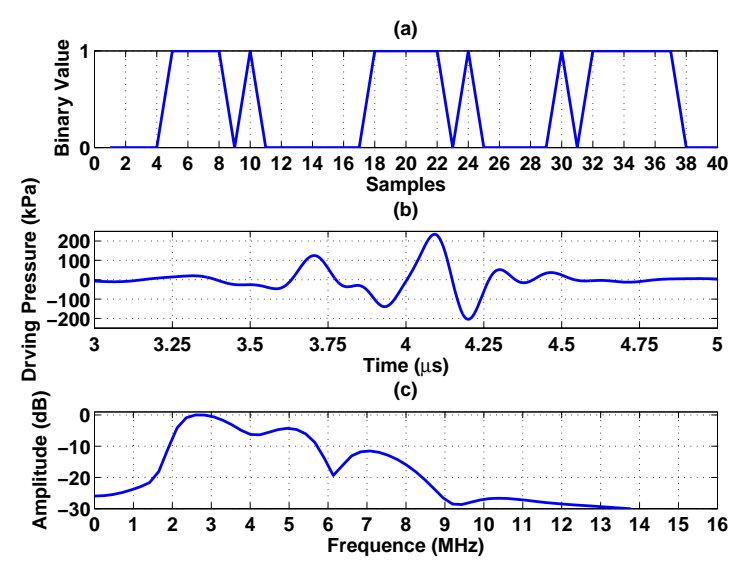

Fig. 3. (a) Optimal binary transmitted signal $x_{1}(n)$ obtained by the genetic algorithm. (b) Signal $p(n)$ at the transducer output (Fig. 1) when $w(n)$ was the optimal binary signal and (c) its spectrum. 


\section{DISCUSSION AND CONCLUSIONS}

Binary sequences were automatically transmitted through a pulse inversion imaging system in order to optimize the CTR frame per frame. This optimization was perfomed without taking into account a priori knowledge of the medium or the transducer. The closed loop system automatically provided an optimal binary command where the $C T R$ was higher than with a fixed-frequency transmitted signal. This optimal setting proposed a filtered binary wave composed of harmonic components and transmitted to the explored medium. These nonlinear components did not damage the contrast, because the pulse inversion properties ensure the extraction of nonlinearities only generated by the medium. This property may be the explanation of the best compromise between the transducer bandwidth and the backscattered responses of microbubbles and tissue.

For future integration in an ultrasound imaging system, the computational complexity was not high. First, the CTR computation from regions of interest ( $L \times L$ size) in the image required $2(2 L+1) 2+1$ operations. Secondly, the genetic algorithm required $0.4\left(12 N_{s}\right)+6$ random selections per generation to achieve the optimum. Taking into account the computing power available for a personal computer, the two last operations must not slow down the optimization process. However, the number of generations to achieve the optimum may be a limiting factor. Since the frame rate can reach 2000 $\mathrm{Hz}$ in some ultrasound scanners, this limitation should be relative. We therefore estimated that the optimization should take less than 5 seconds.

The method could be applied to the ultrasound imaging without using prommable analog transmitter contrary to a transmit frequency optimization. It could open up the optimal command for ultrasound imaging. The next step will be to implement it on an ultrasound scanner. We hope increasing the contrast to help clinician for diagnoses.

\section{REFERENCES}

[1] P. N. Burns, "Instrumentation for contrast echocardiography," Echocardiogr-J Card, vol. 19, no. 3, pp. 241258, Apr. 2002.

[2] F. Forsberg, W. T. Shi, and B. B. Goldberg, "Subharmonic imaging of contrast agents," Ultrasonics, vol. 38, no. 1-8, pp. 93-98, Mar. 2000.

[3] A. Bouakaz, S. Frigstad, F. J. Ten Cate, and N. de Jong, "Super harmonic imaging: A new imaging technique for improved contrast detection," Ultrasound Med Biol, vol. 28, no. 1, pp. 59-68, Jan. 2002.

[4] D. H. Simpson, C. T. Chin, and P. N. Burns, "Pulse inversion doppler: A new method for detecting nonlinear echoes from microbubble contrast agents," IEEE T Ultrason Ferr, vol. 46, no. 2, pp. 372-382, Mar. 1999.
[5] G. A. Brock-fisher, M. D. Poland, and P. G. Rafter, "Means for increasing sensitivity in non-linear ultrasound imaging systems," US Patent 5,577,505, Nov. 1996.

[6] P. Phillips and E. Gardner, "Contrast-agent detection and quantification," Eur Radiol, vol. 14, pp. P4-P10, Oct. 2004.

[7] J. M. G. Borsboom, A. Bouakaz, and N. de Jong, "Pulse subtraction time delay imaging method for ultrasound contrast agent detection," IEEE T Ultrason Ferr, vol. 56, no. 6, pp. 1151-1158, June 2009.

[8] J. M. G. Borsboom, C. T. Chin, A. Bouakaz, M. Versluis, and N. de Jong, "Harmonic chirp imaging method for ultrasound contrast agent," IEEE T Ultrason Ferr, vol. 52, no. 2, pp. 241-249, Feb. 2005.

[9] O. Couture, J. F. Aubry, G. Montaldo, M. Tanter, and M. Fink, "Suppression of tissue harmonics for pulseinversion contrast imaging using time reversal," Phys Med Biol, vol. 53, no. 19, pp. 5469-5480, Oct. 2008.

[10] A. J. Reddy and A. J. Szeri, "Optimal pulse-inversion imaging for microsphere contrast agents," Ultrasound Med Biol, vol. 28, no. 4, pp. 483-494, Apr. 2002.

[11] S. Ménigot, J.-M. Girault, I. Voicu, and A. Novell, “Optimization of contrast to tissue ratio by frequency adaptation in pulse inversion imaging," IEEE T Ultrason Ferr, vol. 59, no. 11, Nov. 2012.

[12] S.-W. Huang and P.-C. Li, "Arbitrary waveform coded excitation using bipolar square wave pulsers in medical ultrasound," IEEE T Ultrason Ferr, vol. 53, no. 1, pp. 106-116, Jan. 2006.

[13] R. J. Eckersley, M.-X. Tang, K. Chetty, and J. V. Hajnal, "Microbubble contrast agent detection using binary coded pulses," Ultrasound Med Biol, vol. 33, no. 11, pp. 1787-1795, Nov. 2007.

[14] R. L. Haupt and S. E. Haupt, Practical Genetic Algorithms, John Wiley \& Sons Inc, Hoboken, NJ, USA, 2 edition, June 2004.

[15] M. E. Anderson, "A 2d nonlinear wave propagation solver written in open-source matlab code," in Proc IEEE Ultras Symp, San Juan, Puerto Rico, Oct. 2000, pp. 1351-1354.

[16] Thomas Szabo, Diagnostic Ultrasound Imaging: Inside Out, Academic Press, London, UK, 2004.

[17] C. Greis, "Technology overview: Sonovue (bracco, milan)," Eur Radiol, vol. 14, pp. P11-P15, Oct. 2004. 
[18] P. Marmottant, S. van der Meer, M. Emmer, M. Versluis, N. de Jong, S. Hilgenfeldt, and D. Lohse, "A model for large amplitude oscillations of coated bubbles accounting for buckling and rupture," J Acoust Soc Am, vol. 118, no. 6, pp. 3499-3505, Dec. 2005.

[19] J. M. Gorce, M. Arditi, and M. Schneider, "Influence of bubble size distribution on the echogenicity of ultrasound contrast agents: A study of sonovue.," Invest Radiol, vol. 35, no. 11, pp. 661-671, Nov. 2000.

[20] J. A. Hossack, P. Mauchamp, and L. Ratsimandresy, "A high bandwidth transducer optimized for harmonic imaging," in Proc IEEE Ultrason Symp, San Juan, Puerto Rico, Oct. 2000, vol. 2, pp. 1021-1024. 